\title{
Curvature radiation in rotating pulsar magnetosphere
}

\author{
P. F. Wang, C. Wang and J. L. Han \\ National Astronomical Observatories, Chinese Academy of Sciences, \\ A20 Datun Road, Chaoyang District, Beijing 100012, China \\ email: pfwang@nao.cas.cn
}

\begin{abstract}
We investigate the curvature radiation from relativistic particles streaming along magnetic field lines and co-rotating with a pulsar. The co-rotation affects the trajectories of the particles and hence the emission properties, especially the polarization. For three density models in the form of core, cone and patches, we calculate the polarized emission at a given height and also the integrated emission for the whole open field line region, and try to explain the generation of circular polarization.
\end{abstract}

Keywords. polarization, radiation mechanisms: nonthermal, pulsars: general

\section{Introduction}

The observed pulsar radio emission are generally highly linearly polarized, and have significant circular polarization (e.g., Lyne \& Manchester 1988; Han et al. 1998; Rankin \& Ramachandran 2003). Various radio emission mechanisms are suggested to explain polarization profiles (e.g., Beskin et al. 1988; Xu et al. 2000; Gangadhara 2010). Curvature radiation serves as one of the most possible emission mechanisms for pulsar radio emission and has been investigated extensively for the coherent emission process, pulsar luminosity, emission spectrum and the polarization features (Buschauer \& Benford 1976; Benford \& Buschauer 1977; Ochelkov \& Usov 1980; Gil \& Snakowski 1990; Gangadhara 2010).

Most previous studies on pulsar curvature radiation ignore the effects of rotation. Relativistic particles in pulsar magnetosphere have co-rotation velocity additional to the streaming velocity along the curved magnetic field lines, which affects the radiation properties. For example, the rotation causes a phase lag between the centers of the position angle curve and the profile (Blaskiewicz et al. 1991). The rotation influences the intensities of the leading and trailing components of the profile (Thomas \& Gangadhara 2007; Dyks et al. 2010).

We depicted the most detailed scenery for pulsar curvature radiation (Wang et al. 2012), especially for the polarized emission features. The emission from particles on the nearby field lines within the $1 / \gamma$ emission cone is considered for a given phase and height. The polarization profiles from a given height and the whole open field line region are calculated for three possible density distributions in the form of core, cone and patches.

\section{Pulsar Curvature Radiation}

For our calculation, we assume co-rotating relativistic particles in the inclined dipole magnetosphere distributed in the form of core, cone and patch, which radiate in curvature radiation mechanism. 


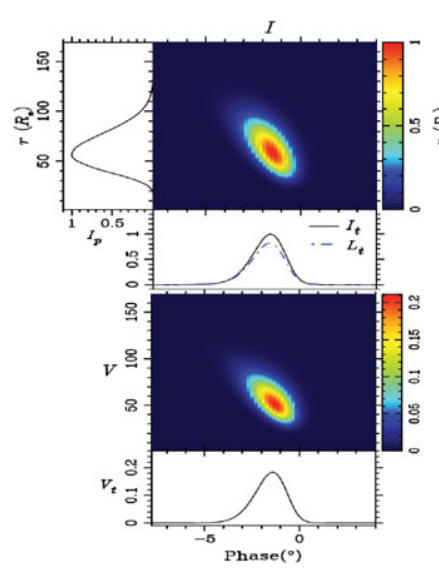

(a) Core Density Model

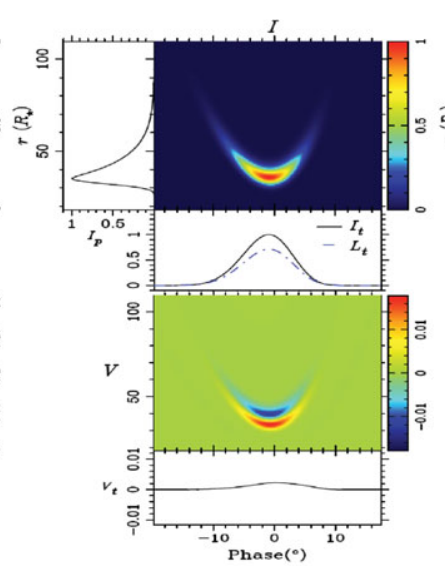

(b) Cone Density Model

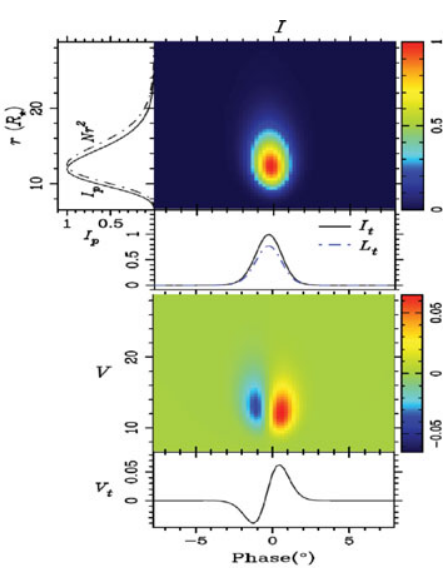

(c) Patch Density Model

Figure 1. Total intensity and circular polarization of the curvature radiation from the core, cone and patch density models, plotted for various phases and heights.

In the co-rotating frame, one relativistic particle or bunch is streaming along a open magnetic field line with velocity $\mathbf{v}^{\prime}$ and acceleration $\mathbf{a}^{\prime}$. The particle trajectory along the magnetic field line is approximated to be circular path in our calculation, because the radiation emitted by an extremely relativistic particle is equivalent to that from a particle moving on an appropriate circular path. The particle also experiences rotation with velocity $\mathbf{v}_{\mathrm{r}}$. In the lab frame, the total velocity, $\mathbf{v}$, should be the addition of $\mathbf{v}^{\prime}$ with $\mathbf{v}_{\mathrm{r}}$ by Lorentz transformation and the total acceleration is the derivative of $\mathbf{v}$. As the radiation of a relativistic particle is beamed in its velocity direction, $\hat{\mathbf{v}}$. The emission location can be determined by requiring the alignment between the wave vector unity $\mathbf{n}$ and $\hat{\mathbf{v}}$. Knowing the velocity, acceleration and emission location of the relativistic particle, we can directly calculate its curvature radiation fields.

The curvature radiation at a given height and rotation phase has the contributions from particles streaming along many nearby field lines around the central tangential point, forming the emission cone of $1 / \gamma$. The total intensity distributes elliptically around the central maximum. The linear polarization has almost the same pattern as total intensity but with a smaller magnitude. The circular polarization has two antisymmetrical lobes with + and - signs corresponding to the left and right hands. Such a polarization pattern of an emission cone is distorted by the additional rotation, more seriously for emission from a larger height.

The observed Stokes parameters of each rotation phase and height are the integration of the entire beam patterns. The particles within the $1 / \gamma$ emission cone may have some kind of density distribution across the field lines. The density gradient across the pattern may result in the net circular polarization. The emission profile is calculated by the integration of the polarized emission at each rotation phase. Due to rotation, the "S" shaped position angle curve is shifted towards a later phase, while the intensity curve to an earlier phase compared to those without rotation. Different density models show different polarization behavior. The particles distributed in density core result in net circular polarization with a single sign for all rotation phases. The emission from a density cone has two components at a large height with sign reversals for circular polarization, but at a lower height they merged to one component with a single sign of circular polarization. For emission from particles in a given density patch, different cuts of a line of sight will cause different profiles. Even when the cuts of a line of sight is fixed, the polarization profiles will be different for various emission heights. 
For a given line of sight, the received emission comes from the tangential points of a range of field lines in a range of heights, and have to be integrated to give the total observed emission for a rotation phase. For the core density model, the circular polarization of the integrated profile has only one single sign (Fig. 1a). The integrated profile from particles in cone density model has insignificant circular polarization due to depolarization (Fig. 1b), and the integrated emission profile in the open field line region from particles in density patches shows the reversal of circular polarization (Fig. 1c).

\section{Conclusion}

We calculated the curvature radiation of particles in the rotating pulsar magnetosphere, and got the following conclusions:

(a) Rotation not only shifts the PA curves along the rotation phase, but also causes an offset, both of which are the first-order functions of the emission height. Their influences on pulsar geometry determination follows the second order functions.

(b) Rotation distorts the patterns for the $1 / \gamma$ emission cone more seriously from larger height and/or smaller impact angle. The density gradients across the patterns will result in the net circular polarization.

(c) For the patch density model, the sense change of circular polarization depends on the impact angle of the sight line. The polarization profiles for a fixed impact angle also vary with emission heights.

(d) The emission from the central peaked density core will usually have significant circular polarization of only one hand. The emission from a density cone has two components at a large height with sign reversals for circular polarization, but at a lower height there is only one component with a single sign of circular polarization.

Our calculations can explain some pulsar polarized emission, but our results can not explain all observational facts. In our model, we did not consider the detailed coherence of the emission and the possible propagation effects. The actual energy and density distributions of particles in the magnetosphere are not clear yet. Furthermore, other emission mechanisms may also work for the observed emission.

\section{Acknowledgments}

This work has been supported by the National Natural Science Foundation of China (11003023 and 10833003).

\section{References}

Benford, G. \& Buschauer R. 1977, MNRAS, 179, 189

Beskin, V. S., Gurevich, A. V., \& Istomin, I. N. 1988, Ap\&SS, 146, 205

Blaskiewicz, M., Cordes, J. M., \& Wasserman, I. 1991, ApJ, 370, 643

Buschauer, R. \& Benford, G. 1976, MNRAS, 177, 109

Dyks, J., Wright, G. A. E., \& Demorest P., 2010, MNRAS, 405, 509

Gangadhara, R. T. 2010, ApJ, 710, 29

Gil, J. A. \& Snakowski, J. K. 1990, A\& A, 234, 237

Han, J. L., Manchester, R. N., Xu, R. X., \& Qiao, G. J. 1998, MNRAS, 300, 373

Lyne, A. G. \& Manchester, R. N. 1988, MNRAS, 234, 477

Ochelkov, I. P. \& Usov, V. V. 1980, Apध̈SS, 69, 439

Rankin, J. M. \& Ramachandran, R. 2003, ApJ, 590, 411

Thomas, R. M. C. \& Gangadhara, R. T. 2007, A\& A, 467, 911

Wang, P. F., Wang, C., \& Han, J. L. 2012, MNRAS, 423, 2464

Xu, R. X., Liu, J. F., Han, J. L., \& Qiao, G. J. 2000, ApJ, 535, 354 